\title{
A Novel Assessment Method of Charging Station Planning Based on Fuzzy Matter Element Theory
}

\author{
Junyi Zhao ${ }^{1}$, Chaoying Yang ${ }^{1}$, Zhiwei Xue ${ }^{1}$ and Yangyang Tan $^{2, *}$ \\ ${ }^{1}$ State Grid Shanxi Electric Power Company, Taiyuan, China \\ ${ }^{2}$ School of Electrical Engineering and Information, Sichuan University, Chengdu, China \\ Corresponding Email: tyy13683448505@136.com
}

\begin{abstract}
Scientific and rational planning of urban electric vehicles (EVs) charging station is an important prerequisite for large scale EVs interact with smart grid friendly. This article realizes the planning assessment of EV charging station based on fuzzy matter element theory. The features of urban EV charging station are analyzed, and the evaluation index system of alternative charging station is established. The paper applies fuzzy matter element analysis method to obtain the optimal fuzzy matter element sequence with alternative points, and as a reference sequence. The weights of alternative points corresponding evaluation index are obtained by entropy method. Then, the paper applies the gray correlation analysis to calculate the gray relational weighing degree of fuzzy matter element sequence of alternative points, and determine the EV charging station plan based on the size of gray relational weighing degree. Finally, the simulation results show that the proposed method is effective and feasible for EV charging station planning.
\end{abstract}

Keywords. Charging station planning, Fuzzy matter element theory, Entropy method, Gray correlation analysis method, Gray relational weighing degree.

\section{Introduction}

In recently years, with the improvement of China's social development and EV ownership increased year by year. The application of EV charging technology is also developing from the "small capacity and small scale" research to the "large capacity and large scale" [1,2]. The development process of EV industry from the world's major countries point of view, EV industry support forces are mainly including government, enterprises, public institutions, research institutions and so on. According to the different participation characteristics of investment operators, charging station operating mode can be divided into government dominant mode, social enterprise dominant mode and user dominant mode. The social enterprise dominant mode is defined as a mode that EV manufacture or social enterprises investing and operating charging station.

The EV charging station planning problem is one of the important research topics in the large-scale application of EVs in cities. Many scholars have carried on massive researches upon the EV charging station planning. Reference [3] proposes an EV charging station planning model in local region considering the load requirements of distribution network and charging requirements of EV users. The model uses the analytic hierarchy process method to determine the weights of charging station alternative points. Reference [4] analyzes impact factors during planning the location of charging station and adopts some indicators that include load ratio, payback period of investment, driven distance and degree of comprehensive satisfaction to evaluate the economy of charging station plan. Reference [5] from the power grid indicators, traffic indicators, economic indicators and planning indicators point of view, basing on the cloud focus theory, the assessment method of charging station planning is conducted. Reference [6] takes into account the cost effectiveness and life cycle cost of EV charging station during charging station operation period. Estimate the capacity of charging station though the traffic flow information and establish the planning model of EV charging station. The objective function of model is maximizing the charging station operation net present value revenue and its constraints including traffic flow of traffic network, power quality of distribution network, economic of power grid and requirements of charging users. Reference [7] studies on the stochastic behavior of electric taxis, takes behavior characteristics of electric taxi and its demand distribution, structure and capacity of distribution network as constraint conditions. At the same time, takes the total cost of charging station construction, equipment maintenance, network loss and charging behavior time as objective function to establish a charging station planning model.

This paper analyzes many factors which influence the planning results of EV charging station and establish evaluation index system of charging station planning. The qualitative analysis and quantitative calculation of the candidate points were carried out and a multidimensional composite fuzzy matter element matrix is established. According to the evaluation method, the degree of membership matrix is obtained and the maximum membership value 
of each evaluation index is selected as the optimal fuzzy matter element sequence. The weighted gray correlation degree of each planning scheme is calculated by combining with entropy weight method and gray relational analysis method. The EV charging station planning program is selected according to the order of relevance degree. Finally, it is verified by simulation analysis and comparison with other methods.

\section{Evaluation index of ev charging station}

Charging station is an energy exchange center for EVs in urban cities and plays a very important role in meeting the needs of EV users. Scientific and reasonable charging station planning program will directly affect the entire city charging service network operating efficiency, and will also affect the urban distribution network and urban traffic safety.

\subsection{Impact Factors Analysis EV Charging Station Planning}

Many factors will affect the EV charging station planning program, that of can be summarized as the following aspects:

1)EV charging station should be built in the main roads or transportation hubs of urban city where the traffic flow is heavy and the consumption of electricity is also large.

2)EV charging station should have a convenient entrance and exit. It can facilitate EV charging the batteries, but also can avoid interfering with urban traffic.

3)The planning scheme of EV charging station not only reach a certain scale to meet the needs of urban charging users, but also control the cost of charging station in a reasonable range in order to achieve better ratio of input-output. It is the premise and basis that can attract more social investors.

4)Distribution of EV charging stations should avoid too concentrated to cause the waste of resources and ensure sufficient numbers of vehicle enter the charging station.

5)EV charging station can be regarded as a large load, charging facilities of charging station should avoid impacting on the grid when they are in operation. In enterprise dominant mode, the EV charging station can be seen as large users. First of all, the grid company will evaluate some indices when the EV charging station access the distribution network, such as harmonic pollution levels, load levels, voltage levels and loss of distribution network. Then, according to the results of the assessment, the grid company requires the EV charging station governing the harmonics until the harmonic meet the national standard. Therefore, charging station investors should focus on the degree of harmonic pollution.

\subsection{Evaluation Index System for EV Charging Station Location}

Considering the social benefit, the economic benefit of the investor and the economic benefit of the charger, the following assessment indices are established:

1) Design scale. Design scale refers to the total charge capacities of charging station, which according to the number of chargers generally.

2) Investment cost. Investment cost refers to the cost of the entire EV charging station, including the cost of land purchase, the cost of charging facilities and transformers purchase, the cost of equipment maintenance, the cost of power loss and the cost of personnel salaries. The total cost can be equivalent to a single charger investment cost by introducing a conversion factor, then, add the fixed investment cost of charging station in actual calculation. Consequantly, the investment cost is a function of the number of chargers.

3) Degree of convenience. Degree of convenience refers to total time of the traveling time and consuming time (including the waiting time and charging time) of EV. The traveling time and consuming time can be expected to get the expected value, the expected value of the sum of the two is the overall time.

4) Adjacent distance. Adjacent distance refers to the shortest distance between new charging station and adjacent charging station.

5) Total vehicle flow. The total vehicle flows refers to the total number of vehicles that passing through the EV charging station in a day.

6) Stop rate. Stop rate refers to the proportion of vehicles that entering the EV charging station in order to charge the batteries.

7) Charging capacity. Charging capacity refers to the single EV charging capacity when EV enters the charging station. Different types of EVs has different charging capacity, even the same type of EVs may also be different. EVs with similar charging capacity can be classified into a category by classification methods and the average amount of capacity is taken for each class of EVs.

8) Harmonic pollution degree. Harmonic pollution degree refers to the total harmonic distortion of the voltage at the point of common coupling when the charging facilities are in operation. This paper focus on harmonic pollution degree which is generated by charging station, whereas harmonic voltage that come from the high voltage and the other harmonic sources as background harmonic [8]. The harmonic pollution degree can be obtained by building simulation model PSCAD software [9]. 


\section{Fuzzy matter element analysis}

\subsection{Multidimensional Complex Fuzzy Matter element Matrix}

Matter element analysis was first proposed by Chinese scholar Cai Wen [10], is a new subject between mathematics and experimental analysis. The basic idea of analysis method is that using three elements "objects, features, values" to describe things, the orderly three elements called matter element, it can be expressed as $R=(M, C, V)$. Supposing that there are $m$ comparison things, each thing has $n$ features, the formula of $n$-dimensional complex fuzzy matter element matrix is:

$$
R=\left[\begin{array}{cccccc} 
& C_{1} & \cdots & C_{j} & \cdots & C_{n} \\
M_{1} & x_{11} & \cdots & x_{1 j} & \cdots & x_{1 n} \\
\vdots & \vdots & \ddots & \vdots & \ddots & \vdots \\
M_{i} & x_{i 1} & \cdots & x_{i j} & \cdots & x_{i n} \\
\vdots & \vdots & \ddots & \vdots & \ddots & \vdots \\
M_{m} & x_{m 1} & \cdots & x_{m j} & \cdots & x_{m n}
\end{array}\right]
$$

where, $M_{i}$ is the $i^{t h}$ evaluation object, $C_{j}$ is the $j^{\text {th }}$ feature of an evaluation index, $x_{i j}$ is the fuzzy value corresponds to the $j^{\text {th }}$ feature of the $i^{\text {th }}$ evaluation object.

\subsection{Membership Matrix}

According to the matter element theory, the membership degree of the fuzzy matter element matrix is obtained by the membership degree calculation method, and then, transform the complex fuzzy matter element matrix into the membership degree matrix. The fuzzy indices are generally divided into positive index, reverse index and fixed constant index. The membership degree calculation of the evaluation indices is also divided into three categories. Basing on the principle of merit, $\mu\left(x_{i j}\right)$ represents the membership degree of the $j^{\text {th }}$ index of $i^{\text {th }}$ evaluation object.

1) Positive index of membership degree

According to the normalization formula of positive index, the value of index is larger, the index is better. The calculation formula is

2)Reverse index of membership degree

$$
\mu\left(x_{i j}\right)=\frac{x_{i j}-\min _{1 \leq i \leq m} x_{i j}}{\max _{1 \leq i \leq m} x_{i j}-\min _{1 \leq i \leq m} x_{i j}}
$$

According to the normalization formula of reverse index, the value of index is smaller, the index is better. The calculation formula is

3)Fixed constant index of membership degree

$$
\mu\left(x_{i j}\right)=\frac{\max _{1 \leq i \leq m} x_{i j}-x_{i j}}{\max _{1 \leq i \leq m} x_{i j}-\min _{1 \leq i \leq m} x_{i j}}
$$
is

where, $c_{0}$ is constant.

According to the membership degree calculation of the above indices, the complex fuzzy matter element matrix $R$ can be transformed into the membership degree matrix $Q$, which is expressed as follows:

$$
Q=\left[\begin{array}{cccccc} 
& C_{1} & \cdots & C_{j} & \cdots & C_{n} \\
M_{1} & \mu\left(x_{11}\right) & \cdots & \mu\left(x_{1 j}\right) & \cdots & \mu\left(x_{1 n}\right) \\
\vdots & \vdots & \ddots & \vdots & \ddots & \vdots \\
M_{i} & \mu\left(x_{i 1}\right) & \cdots & \mu\left(x_{i j}\right) & \cdots & \mu\left(x_{i n}\right) \\
\vdots & \vdots & \ddots & \vdots & \ddots & \vdots \\
M_{m} & \mu\left(x_{m 1}\right) & \cdots & \mu\left(x_{m j}\right) & \cdots & \mu\left(x_{m n}\right)
\end{array}\right]
$$


According to the former calculation method of membership degree, regardless of the original index is a positive index or reverse index or a fixed constant index, the transformation of indices are positive indicators through the equation (2) $\sim$ (4). The optimal fuzzy matter element matrix $Q_{0}$ of optimal evaluation object is constructed by selecting the maximum value of the membership degree of each index, the formula can be expressed as follows:

where, $\mu\left(x_{0 j}\right)=\max \left(\mu\left(x_{1 j}\right), \ldots, \mu\left(x_{i j}\right), \ldots, \mu\left(x_{m j}\right)\right)$.

$$
Q_{0}=\left[\begin{array}{cccccc}
C_{1} & \cdots & C_{j} & \cdots & C_{n} \\
M_{0} & \mu\left(x_{01}\right) & \cdots & \mu\left(x_{0 j}\right) & \cdots & \mu\left(x_{0 n}\right)
\end{array}\right]
$$

\section{Entropy weight method and gray relational analysis method}

\subsection{Entropy weight method}

Entropy originated in thermodynamics, was used to express distribution uniformity of the energy in the space. Entropy theory [11] has been widely used in the field of probability theory, information theory. In order to reflect the importance of each evaluation index, different evaluation indices should be given different weight in the decision-making of EV charging station planning. Entropy weight method [12] is an objective weight method, widely used in the field of index empowerment. According to membership degree matrix, the specific calculation steps that using entropy weight method to calculate the weight is listed as follows:

1)Calculate the entropy of each index, the calculation formula is

$$
H_{j}=-\frac{1}{\ln m} \sum_{i=1}^{m} \frac{\mu\left(x_{i j}\right)+1}{\sum_{i=1}^{m}\left(\mu\left(x_{i j}\right)+1\right)} \ln \left(\frac{\mu\left(x_{i j}\right)+1}{\sum_{i=1}^{m}\left(\mu\left(x_{i j}\right)+1\right)}\right)
$$

2)The weight value of the index is calculated and normalized, the formula is

$$
\omega_{j}=\frac{1-H_{j}}{\sum_{j=1}^{n}\left(1-H_{j}\right)}
$$

\subsection{Gray Relational Analysis}

Gray relational analysis is a kind of evaluation method based on gray system theory ${ }^{[13-14]}$. The basic idea of gray relational analysis is that comprehensively evaluate the things and phenomena which affected by many factors from the holistic view.

According to membership matrix, fuzzy matter element sequences $M_{i}$ and $M_{i}=\left(\mu\left(x_{i 1}\right), \mu\left(x_{i 2}\right), \ldots, \mu\left(x_{i n}\right)\right)$. According to the optimal fuzzy matter element matrix, an optimal fuzzy matter element sequence can be obtained as $M_{0}=\left(\mu\left(x_{01}\right)\right.$, $\left.\mu\left(x_{02}\right), \ldots, \mu\left(x_{0 n}\right)\right)$. In this paper, the sequence $M_{0}$ as a reference sequence, basing on the theory of gray relational analysis, the corresponding coefficient of element which comparing sequence $M_{i}$ with sequence $M_{0}$ is calculated as follows:

$$
\xi_{i j}=\frac{\min _{i}\left|\mu\left(x_{0 j}\right)-\mu\left(x_{i j}\right)\right|+\beta \max _{i}\left|\mu\left(x_{0 j}\right)-\mu\left(x_{i j}\right)\right|}{\left|\mu\left(x_{0 j}\right)-\mu\left(x_{i j}\right)\right|+\beta \max _{i}\left|\mu\left(x_{0 j}\right)-\mu\left(x_{i j}\right)\right|}
$$

where, $\xi_{i j}$ is correlation coefficient of the $i^{\text {th }}$ evaluation object corresponds to the $j^{\text {th }}$ evaluation index. $\beta$ is resolution factor, $0<\beta<1$, the value of $\beta$ is smaller, the greater the difference between the factors, the ability to distinguish is stronger, and value of $\beta$ is usually taking 0.5 .

The factors that influence the location decision of EV charging station are different in the comprehensive evaluation. Therefore, the influence factor weight coefficient is introduced, and then the weight gray incidence degree $D_{i}$ of each fuzzy matter element sequence and the optimal fuzzy matter element sequence of the $i^{\text {th }}$ evaluation object is calculated, the calculation formula is

$$
D_{i}=\sum_{j=1}^{n} \omega_{j} \xi_{i j}
$$

The weight gray correlation degree is sorted by increase order. The value is larger, the more consideration will be given to the factors that influence the planning decision-making of EV charging station planning scheme, and the more it meets the actual planning requirements of EV charging station.

\section{Case studies}


In this section, case studies under different scenarios are conducted to demonstrate the proposed method.

A power grid company plans to build a new EV charging station around the beltway in a city. According to the actual market strategy and distribution network layout, at the same time, considering the traffic flows, site locations and surroundings, the power grid company proposing 4 sites as EV charging station candidates.

In terms of investment cost of EV charging station, assume that the investment cost is a linear function of the number of chargers and the unit price of one charger is $100,000 \$$. After the actual inspection of candidates and comprehensive evaluation of the industry experts, the quantification value of each evaluation index of candidates can be listed in Table 1.

Table 1. Quantized values of evaluation index for EV charging station alternative points around the beltway.

\begin{tabular}{ccccccccc}
\hline Candidates & $\begin{array}{c}\text { Design } \\
\text { scale }\end{array}$ & $\begin{array}{c}\text { Investment } \\
\text { cost/10 } \$\end{array}$ & $\begin{array}{c}\text { Convenience } \\
\text { degree/min }\end{array}$ & $\begin{array}{c}\text { Adjacent } \\
\text { distance/km }\end{array}$ & $\begin{array}{c}\text { Total } \\
\text { vehicle } \\
\text { flow }\end{array}$ & $\begin{array}{c}\text { Stop rate } \\
/ \%\end{array}$ & $\begin{array}{c}\text { Charging } \\
\text { capacity } \\
/ \text { KW·h }\end{array}$ & $\begin{array}{c}\text { Harmonic } \\
\text { pollution } \\
\text { degree/\% } / \%\end{array}$ \\
\hline 1 & 12 & 180 & 38 & 4.0 & 6700 & 3.6 & 46 & 2.8 \\
2 & 13 & 185 & 30 & 3.6 & 7400 & 2.9 & 52 & 3.1 \\
3 & 11 & 160 & 40 & 2.8 & 6200 & 4.0 & 51 & 3.6 \\
4 & 15 & 205 & 32 & 5.1 & 7900 & 2.8 & 47 & 2.9 \\
\hline
\end{tabular}

The multidimensional complex fuzzy matter element matrix $R$ can be expressed as follows.

$$
R=\left[\begin{array}{llllllll}
12 & 180 & 38 & 4.0 & 0.67 & 3.6 & 46 & 2.8 \\
13 & 185 & 30 & 3.6 & 0.74 & 2.9 & 52 & 3.1 \\
11 & 160 & 40 & 2.8 & 0.62 & 4.0 & 51 & 3.6 \\
15 & 205 & 32 & 5.1 & 0.79 & 2.8 & 47 & 2.9
\end{array}\right]
$$

The size of EV charging station must meet the public EV charging requirement, not the bigger the better, but estimating the total charging power according to the current charge needs, therefore, the EV charging station size index belonging fixed constant index and the numbers of charger defining as 13 . The lower of the investment cost, the more attractive for investors. The shorter for EV charging users reaching the charging station, the more charging users choose the charging station for charge. The smaller for EV charging station produce harmonic pollution, the bigger of investment benefit. Thus, the investment cost index, the convenience degree index and the harmonic pollution degree index is explained above are belonging reverse index.

The farther away from the adjacent charging station, the more charging users will be attracted and charge the battery in this EV charging station. The bigger for traffic flow ahead of the charging station, stop rate and charging capacity of single EV, the bigger for investment benefit of EV charging station. As a result, the 3 indices above are belonging positive index.

According to the formula (2), (3) and (4), the transpose matrix of membership matrix $Q^{T}$ is expressed as follows:

The optimal fuzzy matter element matrix $Q_{0}$ is obtained by formula (6).

$$
Q=\left[\begin{array}{cccccccc}
0.923 & 0.556 & 0.200 & 0.522 & 0.294 & 0.667 & 0 & 1.000 \\
1.000 & 0.444 & 1.000 & 0.348 & 0.705 & 0.083 & 1.000 & 0.625 \\
0.846 & 1.000 & 0 & 0 & 0 & 1.000 & 0.833 & 0 \\
0.867 & 0 & 0.800 & 1.000 & 1.000 & 0 & 0.167 & 0.875
\end{array}\right]
$$

$$
Q_{0}=\left[\begin{array}{llllllll}
1.0 & 1.0 & 1.0 & 1.0 & 1.0 & 1.0 & 1.0 & 1.0
\end{array}\right]
$$

Firstly, the weight after normalization process and the correlation coefficient of each evaluation index is calculated by formula (7) to (10). Secondly, the degree of combined weighted gray relational of each candidate is calculated by formula (11). The calculation results are listed in Table 2.

Table 2. Gray relational weighing degree of alternative points.

\begin{tabular}{ccccc}
\hline$i$ & 1 & 2 & 3 & 4 \\
\hline$D_{i}$ & 0.5274 & 0.6399 & 0.5958 & 0.6302 \\
\hline
\end{tabular}

According to Table 2, gray relational weighing degree of candidate 2 is larger than the other EV charging station candidates significantly. According to the evaluation indices are listed in Table 1, in terms of operator, they can reduce the investment cost by means of competitive negotiation, they can improve the stop rate by means of charging price concessions, and they can heighten the charging capacity of single EV by using the advanced charging technologies for chargers.

Basing on the deep analysis above, in this section, we choose candidate 2 for example, alter the indices including investment cost of charging station, stop rate and charging capacity of single EV and analyze how the index influence on gray relational weighing degree. The analysis results are described in Figure 1. 


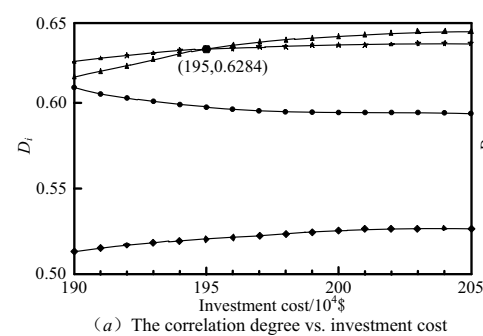

(a) The correlation degree vs. investment cost

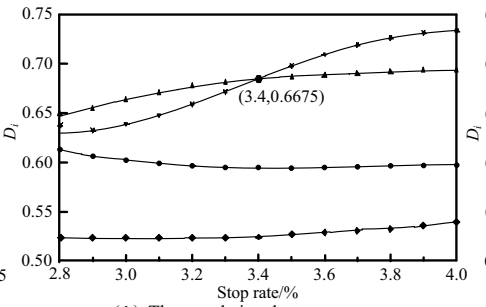

(b) The correlation degree vs. stop rate $\bullet D_{1}+D_{2} \rightarrow D_{3} \leftarrow D_{4}$

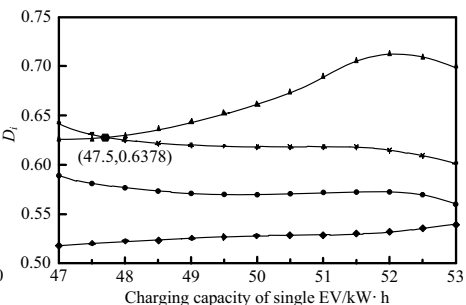

(c) The correlation degree vs. Charging capacity of single EV

Fig.1. The relationship between gray relational weight degree of candidates with evaluation index.

According to the analysis results in Figure 1 (a), gray relational weighing degree of candidate 2 and 4 are reducing gradually by cutting down the investment cost of charging station. The gray relational weighing degree of candidate 2 is greater than candidate 4 when the investment cost of candidate 4 below 1,950,000\$, at the same time, candidate 2 is regards as new charging station planning site. The analysis results in Figure 1 (b) show that investors improve the stop rate by adopting the effective incentive strategies or preferential measures can also achieve similar result, consequently, candidate 2 is replaced by candidate 4 when the stop rate is greater than $3.4 \%$. Simultaneously, basing on the Figure 1 (c), the operators adopts the advanced charging technologies for charging, not only saving the charging time of EV, but also improving the charging capacity of single EV. We can see that candidate 2 is replaced by candidate 4 when the charging capacity of single EV is greater than $47.5 \mathrm{~kW} \cdot \mathrm{h}$.

It can be seen that the various evaluation indices of charging stations will have a greater impact on the final EV charging station planning scheme.

\section{Conclusion}

In this paper, an evaluation method of EV planning scheme based on fuzzy matter element theory is proposed for the existing charging station planning problem. It is found that the proposed method can find the final planning scheme conveniently by case study 1 . By analyzing the impact of variable parameters such as investment cost, stop rate and charging capacity of single EV on the planning results, operators can adopt scientific and reasonable incentive measures to adjust the planning results and increase the investment benefit. Finally, the results of case study 2 demonstrate the feasibility and effectiveness of the proposed method by comparing it with other methods.

\section{References}

1. Fakhrazari, H. Vakilzadian, and F. F. Choobineh, "Optimal energy scheduling for a smart entity," IEEE Transaction on Smart Grid, vol. 5, pp. 2919-2928, 2014.

2. K. S. Reddy, L. K. Panwar, R. Kumar, and B. K. Panigrahi, "Distributed resource scheduling in smart grid with electric vehicle deployment using fireworks algorithm." Journal of Modern Power Systems and Clean Energy, vol. 4, pp. 1-12, 2016.

3. $\quad$ L. F. Kou, Z. F. Liu, and H. Zhou, "Modeling algorithm of charging station planning for regional electric vehicle." Modern Electric Power, vol. 27, pp. 44-48, 2010.

4. C. Zhang, and H. Teng, "Planning model and evaluation method for electric vehicle charging station." Proceedings of the CSU-EPSA, vol. 26, pp. 49-52, 2014.

5. Z. J. Peng, J. Lan, Y. Chen, Q. Zou, L. G. Jia, and J. Yang, "EV charging station sitting method based on cloud focus theory." Electric Power Construction, vol. 36, pp. 1-7, 2015.

6. X. Q. Huang, H. Yang, J. Chen, L. Jiang, and Y. J. Cao, "Optimal planning of electric vehicle charging stations based on life cycle cost and quantum genetic algorithm." Automation of Electric Power Systems, vol. 39, pp. 176$182,2015$.

7. S. Y. Ge, R. Li, J. Han, H. Liu, T. Li, and H. H. Lian, "Charging station planning considering probability behavior characteristic of electric taxi." Automation of Electric Power Systems, vol. 40, pp. 50-58, 2016.

8. X. Zhao, and H. G. Yang, " A new method to calculate the utility harmonic impedance based on FastICA." IEEE Transactions on Power Delivery, vol. 31, pp. 381-388, 2016.

9. N. Li, M. Huang, "Analysis on harmonics caused by connecting different types of electric vehicle chargers with power network." Power System Technology, vol. 35, pp. 170-174, 2011.

10. D. K. W. Ng, and W. Cai, "Treating non-compatibility problem from matter element analysis to extenics." Acm Sigice Bulletin, vol. 22, pp. 2-9, 1997. 
11. R. Li, Z. Zhao, X. Zhou, and J. Palicot, "The prediction analysis of cellular radio access network traffic: from entropy theory to networking practice." IEEE Communications Magazine, vol. 52, pp. 234-240, 2014.

12. H. Deng, X. Sun, M. Liu, C. Ye, and X. Zhou, "Infrared small-target detection using multiscale gray difference weighted image entropy." IEEE Transactions on Aerospace and Electronic Systems, vol. 52, pp. 60-72, 2016.

13. J. Wang, J. Wang, T. Zhang, Y. Wu, "Probability estimation based on grey system theory for simulation evaluation." Journal of Systems Engineering and Electronics, vol. 27, pp. 871-877, 2016.

14. L. Chen, B. Tian, W. Lin, and B.Ji, "Analysis and prediction of the discharge characteristics of the lithium-ion battery based on the grey system theory." IET Power Electronics, vol. 8, pp. 2361-2369, 2015. 